\title{
Stoma Site Infection, CTCAE
}

National Cancer Institute

\section{Source}

National Cancer Institute. Stoma Site Infection, CT CAE. NCI Thesaurus. Code C143860.

A disorder characterized by an infectious process involving a stoma (surgically created opening on the surface of the body). 